\title{
Grand Rounds Panel Discussion: Blood Thinners Peri-Operatively: What to do?
}

\author{
Jad Al Danaf, MD \\ Thomas Jefferson University, jad.aldanaf@jefferson.edu \\ Anusha Govind, MD \\ Thomas Jefferson University, anusha.govind@jefferson.edu \\ Loheetha Ragupathi, MD \\ Thomas Jefferson University, Loheetha.ragupathi@jefferson.edu \\ Shuwei Wang, MD \\ Thomas Jefferson University, shuwei.wang@jefferson.edu
}

Follow this and additional works at: https://jdc.jefferson.edu/tmf

Part of the Internal Medicine Commons

Let us know how access to this document benefits you

\section{Recommended Citation}

Al Danaf, MD, Jad; Govind, MD, Anusha; Ragupathi, MD, Loheetha; and Wang, MD, Shuwei (2016) "Grand Rounds Panel Discussion: Blood Thinners Peri-Operatively: What to do?," The Medicine Forum: Vol. 17 , Article 21.

DOI: https://doi.org/10.29046/TMF.017.1.021

Available at: https://jdc.jefferson.edu/tmf/vol17/iss1/21

This Article is brought to you for free and open access by the Jefferson Digital Commons. The Jefferson Digital Commons is a service of Thomas Jefferson University's Center for Teaching and Learning (CTL). The Commons is a showcase for Jefferson books and journals, peer-reviewed scholarly publications, unique historical collections from the University archives, and teaching tools. The Jefferson Digital Commons allows researchers and interested readers anywhere in the world to learn about and keep up to date with Jefferson scholarship. This article has been accepted for inclusion in The Medicine Forum by an authorized administrator of the Jefferson Digital Commons. For more information, please contact: JeffersonDigitalCommons@jefferson.edu. 


\title{
Blood Thinners Peri-Operatively: What to do?
}

\author{
Jad AL Danaf, MD, MPH (Facilitator), Anusha Govind, MD, Loheetha Ragupathi, MD, and Shuwei Wang, MD
}

\section{PANELISTS}

Mathew DeCaro, MD: Associate Professor of Cardiology, Director of the Coronary Cardiac Unit, Cardiology fellowship program director

John Doherty, MD: Professor of Cardiology

Gregary Markefka, MD: Associate Professor of Cardiology, Associate Director of the Coronary Cardiac Unit, Cardiology fellowship assistant program director

Geno Merli, MD: Professor of Vascular Medicine, Co-director of Jefferson Vascular Center

Srikanth Nagalla, MD, MS: Associate Professor of Medicine, Director, Clinical Hematology

Eric Schwenk, MD: Assistant Professor of Anesthesiology, Director of orthopedic anesthesia

Barry Ziring, MD: Clinical Associate Professor of Internal Medicine / Primary Care, Director of the division of Internal Medicine

\section{INTRODUCTION}

The population size today is increasing and becoming more of an aging population. This carries with it a package of chronic illnesses associated with aging and particularly the need for surgeries.

Atrial fibrillation is the most common arrhythmia causing approximately $20 \%$ of ischemic stroke cases with estimated annual costs of $\$ 6$ billion in the United States ${ }^{1,2}$ Many patients undergoing surgeries are on blood thinning agents; either oral antiplatelet (AP) for established coronary artery disease of anticoagulants (AC) for atrial fibrillation or thromboembolic disease, putting them at risk of bleeding. It is estimated that about $5-15 \%$ of patients undergoing coronary stent implantation are expected to undergo a surgical procedure within 2 years ${ }^{3}$.

Hence, it is of utmost importance to be familiar with the perioperative management of $A P$ and $A C$ agents to balance the risks of thromboembolic events, ischemic cardiac events and bleeding. The key to such management is extensive benefit-risk discussions with patients and seamless coordination within a multidisciplinary team of surgeons, interventional proceduralists, anesthesiologists, hematologists, vascular medicine specialists, cardiologists, primary care physicians and nurses. We present a case based multidisciplinary panel discussion to facilitate better understanding of this topic.
Before starting the case discussions, here are some definitions with respect to AC and AP agents

- Interrupt: Omit one or more doses of the agent, based on the bleeding risk

- Bridge: Substitute the oral agent with a parenteral agent, based on the thrombotic risk

- Elective surgery: Can/should be delayed until the patient is medically stable.

- Urgent surgery: Needs to be done within 48 hours, needs quick attention and can be delayed for medical stability. This includes oncologic surgeries

- Emergent surgery: Needs to be done immediately, otherwise the patient will die.

The general approach to the perioperative management of blood thinners depends on 4 major steps

1. Estimating thromboembolic or ischemic risk

2. Estimating the bleeding risk (surgical, anesthesia type and patient factors)

3. Deciding whether and when to interrupt the AC or AP agents

4. Deciding whether there is a need for bridging therapy until the oral agent can be resumed

\section{PERIOPERATIVE MANAGEMENT OF AC AGENTS}

A modified risk stratification by Douketis et al of the consensus statement on the perioperative management of antithrombotic therapy is presented in Table 1 as published in 2012 4 . Estimating the thromboembolic risk depends on three main illness categories: presence of a mechanical heart valve, atrial fibrillation and venous thromboembolic disease. Patients at very high risk of thromboembolic phenomena should either be continued on their AC agents perioperatively or bridged with a parenteral agent. Patients with low to moderate risk of thromboembolic phenomena can be safely managed perioperatively off their $A C$ agents in case of high risk of bleeding that necessitates cessation of the AC agent.

The thrombotic risk drives the decision for bridging and the bleeding risk drives the decision for $A C$ interruption. A risk-benefit ratio will dictate the most suitable plan of action in every patient, and in most scenarios, these decisions differ from case to case. 
Table 1: Estimates of Thromboembolic Risks

\begin{tabular}{|c|c|c|c|}
\hline Risk Category & Mechanical valve & $\mathrm{AF}$ & VTE \\
\hline Very high & $\begin{array}{l}\text { - } \text { MV prosthesis } \\
\text { - Cage-ball or tilting disc AV } \\
\text { - Stroke/TIA: } 6 \text { months }\end{array}$ & $\begin{array}{l}\text { - CHA2DS2-VASc 6-9 } \\
\text { - Stroke/TIA: } 3 \text { months } \\
\text { - Rheumatic valve }\end{array}$ & $\begin{array}{l}\text { - VTE: } 3 \text { months } \\
\text { - Severe thrombophilia } \\
\text { (protein C or S deficiency, } \\
\text { AT III deficiency, APLS) }\end{array}$ \\
\hline High & $\begin{array}{l}\text { Bileaflet AV + : AF, stroke/TIA, } \\
\text { HTN, DM, CHF, >75 years old }\end{array}$ & CHA2DS2-VASC 4-5 & $\begin{array}{l}\text { - VTE: 3-12 months } \\
\text { - Thrombophilia: FV-Leiden, } \\
\text { prothrombin gene mutation } \\
\text { - Recurrent VTE } \\
\text { - Active Cancer }\end{array}$ \\
\hline Low-moderate & Bileaflet AV + No other risk factors & $\begin{array}{l}\text { CHA2DS2-VASc 2-3, } \\
\text { no TIA/Stroke }\end{array}$ & VTE $>12$ months \\
\hline
\end{tabular}

AF: atrial fibrillation; VTE: venous thromboembolism; MV: mitral valve; AV: aortic valve; TIA: transient ischemic attack; HTN: hypertension; DM: diabetes mellitus; CHF: congestive heart failure; AT: antithormbin; APLS: anti phospholipid syndrome; FV: factor five. Modified from Douketis JD et al. ${ }^{4}$

\section{Table 2: Estimates of Bleeding Risk}

\section{Risk category}

- High risk* (cardiac, vascular, general surgery, kidney biopsy, polypectomy, bilateral knee, laminectomy, neurosurgery, urological)

Type of Surgery

- Low risk** (eye, cholecystectomy, hysterectomy, tooth extraction, endoscopy+biopsy, pacemaker, bronchoscopy + biopsies, knee, hip, arthroscopy)

Patient factors

- HAS-BLED score (hypertension, abnormal renal or liver function (two points for both), stroke, bleeding tendency, labile INRs, elderly age, and antiplatelet drugs or alcohol). Total 9 points.

- High risk: $>3$ points (HR 11.8, 95\% Cl 5.6-24.9) according to the BORDER registry

HR: Hazard ratio; *A high risk of bleeding is defined as a 2-4\% risk of a major bleed within 2 days post-operatively; ${ }^{*} \mathrm{~A}$ low risk of bleeding is defined as a $0-2 \%$ risk of a major bleed within 2 days post-operatively; Data from Spyropoulos et al ${ }^{6}$.

Table 3: Bridging Therapy Management Peri-Operatively.

\begin{tabular}{|c|c|c|}
\hline Bleeding risk & Pre-operative & Post-operative \\
\hline Low & $\begin{array}{l}\text { - Start on day-3 when stopped Warfarin day }-5 \\
\text { - Omit dose on day } 0 \\
\text { - Omit evening dose day }-1 \text { if BID dose } \\
\text { - } 50 \% \text { total dose if OD day-1 }\end{array}$ & $\begin{array}{l}\text { Resume therapeutic dose day } 1 \text {, if } \\
\text { hemostasis is secure }\end{array}$ \\
\hline High & $\begin{array}{l}\text { - Start on day-3 when stopped Warfarin day }-5 \\
\text { - Omit dose on day } 0 \\
\text { - Omit evening dose day }-1 \text { if BID dose } \\
\text { - } 50 \% \text { total dose OD day }-1\end{array}$ & $\begin{array}{l}\text { Resume therapeutic dose days } 2-3 \text { if } \\
\text { hemostasis is secure } \\
\text { OR } \\
\text { Low dose LMWH ( } 30 \text { or } 40 \mathrm{mg} \text { ) when } \\
\text { hemostasis is secure }\end{array}$ \\
\hline
\end{tabular}

BID: twice a day; OD: once a day; TIA: transient ischemic attack; Data from Douketis et al4, Douketis et al7, Hirsh et al 8 . 


\section{Al Danaf, MD et al.: Grand Rounds Panel Discussion: Blood Thinners Peri-Operatively: What to do?}

The bleeding risk is estimated based on surgical risk and patient's risk. A major bleed is generally defined as bleeding that is fatal, intracranial, requires surgery to correct, lowers the hemoglobin by $\geq 2 \mathrm{~g} / \mathrm{dL}$, or requires transfusion of $\geq 2$ units of packed red blood cells.

The goal of bridging is to minimize the patient's time being off the oral anticoagulant for more protection against thromboembolic events. The commonly used agents are subcutaneous enoxaparin or parenteral unfractionated heparin which have similar safety profiles and efficacy. However, the risk of bleeding should be also factored in while deciding when to start and stop the bridging agent. The available consensus statements on bridging management are summarized in table ${ }^{34,7.8}$

To note, most of the above recommendations are either class $2 \mathrm{~b}$ or $2 \mathrm{c}$ recommendations, and cannot be considered guidelines as per the definition of guidelines; rather they reflect consensus statements based on the published available body of evidence.

The following are transcription of case discussions among the different experts regarding their recommendations for perioperative management of each patient.

\section{Case 1: Warfarin}

A 76-year-old female with non-valvular atrial fibrillation, hypertension, and prior stroke six months ago, receiving warfarin, requires elective hip replacement with neuraxial anesthesia; renal function is normal, and weight is $75 \mathrm{~kg}$

$\mathrm{CHA} 2 \mathrm{DS} 2-\mathrm{VASC}=6 \rightarrow$ Very high risk of thrombosis and high risk of bleeding

Dr. DeCaro: I tend to treat patients with a previous stroke aggressively irrespective of the time frame, so I would bridge this patient perioperatively.

Dr. Shwenck: We leave the decision to the orthopedic surgeons to determine the urgency of the procedure and if it can be delayed. We will still follow the currently available guidelines particularly concerning bridging.

Dr. DeCaro: We would also stop heparin at midnight prior to the procedure.

Dr.Merli: There is a study in Annals of Internal Medicine that showed no anti-Xa effect on the morning of the surgery if the enoxaparin is stopped 24 hours prior to surgery.

Audience: When we try to risk assess, we multiply the likelihood by the impact of an illness. For example, if 100 people had something done and $98 \%$ will do well, that's a great likelihood ration. The impact of having something go bad, such as paralysis can affect the remaining $2 \%$. Therefore, when we individualize care, patients should understand the risks that they are agreeing to and quoting likelihood of success is less meaningful that weighing both the likelihood of success and impact of failure.

Dr. Merli: I don't think any orthopedic surgeon would allow their patients to receive a full dose of low molecular weight heparin (LMWH) on the same day after surgery, so they might recommend either lower doses or prophylactic doses.

Dr. Doherty: There was a survey that was recently sent out to the ACC members and asked:" Does your institution have a well structured process on how to manage perioperatively?"; and only one percent said they had a comprehensive well structured system on how to manage these patients perioperatively.

Audience: Can the panel comment on the appropriate time to stop heparin prior to surgery? We have had internal differences in opinions and recommendations.

Dr. Nagalla: The ACCP guidelines recommend stopping heparin 4-6 hours prior to surgery. For LMWH, the recommendation is at least 24 hours $^{4}$. There are also some situations when we may need to use $40 \mathrm{mg}$ of enoxaparin instead of a therapeutic dose 24 hours prior to surgery in cases of high risk of bleeding in need for bridging therapy.

\section{Suggested management plan for case 1:}

- Stop warfarin day-5

- Preoperative bridging with LMWH day-3, with last dose on the morning of day-1.

- Resume warfarin within 24 hours after surgery (usual dose).

- Postoperative low dose LMWH for VTE prevention within 24 hours after surgery until postoperative bridging is started.

- Postoperative bridging on postoperative day 2 or 3 , when hemostasis is secured; continue for at least 4-5 days, until the INR is therapeutic.

\section{Case 2: Rivaroxaban}

A 68-year-old female with non-valvular atrial fibrillation, hypertension, and congestive heart failure, receiving rivaroxaban $15 \mathrm{mg}$ daily in the morning, requires a dental cleaning and two dental extractions; $\mathrm{CrCl}$ is $35 \mathrm{~mL} / \mathrm{min}$.

CHA2DS2-VASC score $=4 \rightarrow$ high risk of thrombosis and low risk of bleeding

Dr. Merli: I would not bridge this patient and would stop rivaroxaban. She is on the lower dose, so the question is when to stop it. ASRA guidelines ${ }^{10}$ are not for dental procedures. Within 48 hours ( 4 half lives), rivaroxaban should be cleared, and 3 days for Xa inhibitors. I would restart rivaroxaban 24 hours after the procedure, and would think of an alternative drug for the future due to her low $\mathrm{CrCl}$, such as warfarin.

Dr. Nagalla: I would treat her differently; I would not stop rivaroxaban and would use local pro-hemostatic agents such as tranexamic acid mouthwash. Hold dose on day of procedure, restart with next dose.

Dr. Ziring: In the community they are less tolerant of bleeding so we tend to stop the oral AC sooner. 
Dr. Doherty: This would be a proceed-uninterrupted plan if a patient is taking Warfarin. The answer of this case might be different in 5 years due to the expanding data of direct acting anticoagulants (DOACS). Shall we delay the morning dose or omit the dose on that day?

\section{Suggested management plan for case 2:}

- Stop rivaroxaban on the day of the procedure.

- Use oral tranexamic acid mouthwash just before the procedure and two to three times that day after the procedure.

- Resume rivaroxaban the day after the procedure, after at least 24 hours have elapsed (assuming the dental extractions were uneventful).

\section{Case 3: Apixaban}

A 55 year old male with an unprovoked deep vein thrombosis (DVT) four months ago, receiving apixaban $5 \mathrm{mg}$ twice daily, who requires a colonoscopy because of a personal history of premalignant colorectal polyps with planned polypectomy; renal function is normal.

$\rightarrow$ high risk of thrombosis and high risk of bleeding

Dr. Nagalla: It was noticed that the highest risk of thrombosis recurrence is within 3 months of the VTE. In this case, since the DVT is $>3$ months ago, we would focus more on the bleeding risk as compared to the thrombotic risk; Stopping Apixaban 48 hours prior to the procedure is reasonable, with no bridging, and resuming Apixaban after 24 hours. We can also give them $2.5 \mathrm{mg}$ of Apixaban 12 hours after the procedure then resume full dose 24 hours after the procedure.

Dr. Merli: I agree with Dr. Nagalla, I would stop it 48 hours prior to the procedure. The guidelines from ASRA are for regional anesthesia, so we have to be careful in interpreting these results.

Audience: How would the answer change if the case is of a PE instead of a DVT?

Dr. Nagalla: Depends on the severity of a PE: Hemodynamic stability and if within the past 3 months, need for thrombolysis...etc. So even for PE, same risk stratification should take place.

Dr. Doherty: This case can be divided to what you do pre and postop, because with a normal renal function, you can hold one dose of Apixaban, emphasizing that you almost never bridge patients on DOACs. If it ends up being a large polyp, or a tiny polyp or no polyp, then post procedurally management differs.

\section{Suggested management plan for case 3:}

- Stop apixaban day-2

- No bridging

- Resume apixaban days 2-3 after the polypectomy/ colonoscopy.

\section{Case 4: Warfarin}

A 69-year-old male with chronic atrial fibrillation and hypertension on Warfarin, requires a Whipple surgery for pancreatic adenocarcinoma.

CHA2DS2-VASC score $=2 \rightarrow$ low-moderate risk of thrombosis and high risk of bleeding

Dr. Ziring: Moderate risk is based on CHA2DS2-VASc risk but this patient has other risks of thrombosis: cancer and undergoing a major surgery. Therefore here are two approaches:

- Not bridge and restart coumadin + Sub-cutaneous heparin DVT prophylaxis

- LMWH before surgery, discontinue it prior to surgery, after surgery restart $\mathrm{LMWH}$ at lower dose, increase to full dose at time of discharge. Removes this confusion of pills and bridging.

Dr.Doherty: If the patient is ambulatory, the thrombotic risk is acceptably low. Risk is not stroke or systemic embolism; rather it is mainly of DVT. Therefore, post-op needs prophylactic dose prior to full dose enoxaparin or warfarin.

Dr. DeCaro: So post-op, transitioning to Warfarin, I would give DVT prophylaxis until INR is therapeutic on Warfarin.

Dr. Nagalla: There is an advantage of Warfarin post-op. Bowel resection; the advantage of using warfarin is that we have an INR to monitor as compared to DOACs. Patients on DOACs were excluded from most studies.

\section{Suggested management plan for case 4:}

- Stop Warfarin day-5

- No bridging

- Resume Warfarin day of surgery.

- Use sub-cutaneous heparin or LMWH for DVT prophylaxis on day of surgery onwards until INR is therapeutic

\section{Case 5: Rivaroxaban and regional anesthesia}

A 65 yo male with history of AF and hypertension taking rivaroxaban presents for primary total hip replacement. He denies any history of diabetes, coronary artery disease, stroke, or heart failure. Hemoglobin 14.0.

CHA2DS2-VASC score $=2 \rightarrow$ low-moderate risk of thrombosis and low risk of bleeding

Dr. Schwenk: This is a common case. First thing, what is the bleeding risk? Primary hip replacement is important to distinguish from revision or more complicated hip surgeries. Thus the bleeding risk falls into the intermediate bleeding risk category. Second thing, what is the surgeon's and anesthesiologist's preference? We have an institutional practice that the surgeons prefer regional anesthesia, but I believe that the benefits of neuraxial anesthesia is exaggerated since most of the data is based on retrospective 


\section{Al Danaf, MD et al:: Grand Rounds Panel Discussion: Blood Thinners Peri-Operatively: What to do?}

data analysis. Third question is whether we need to bridge or not? And with what agent?

ASRA are working on the 4th edition ${ }^{11}$ of anticoagulation management perioperatively and they published a draft table highlighting the recommended time intervals before and after neuraxial block or epidural catheter removal.

Dr. Merli: I would stop rivaroxaban for 2 days and restart it in the orthopedic dose of $10 \mathrm{mg}$ post procedurally, whenever the surgeons are ok with us restarting it. If anesthesiologists prefer 3 days, I would be ok with it as well. When do we go to the regular dose? Cardiologists may say in 2 weeks post-op? But we don't know what the correct answer is. Some surgeons might even object to using rivaroxaban post-op. Should we use Warfarin instead? Or LMWH? These are nebulous questions. I would not bridge upfront and would restart rivoraxaban post-op.

\section{Suggested management plan for case 5:}

- Stop Rivaroxaban day-3 to reduce risk of spinal hematoma (may need longer with impaired $\mathrm{CrCl}$ or other agents that increase bleeding risk)

\section{- No Bridging}

- Resume Rivaroxaban day +1 , if team prefers to delay, consider LMWH or SQH for DVT prophylaxis

Figure 1. Algorithm for perioperative management of antiplatelet therapy. Adapted from Di Minno et al ${ }^{13}$

ADP: adenosine diphosphate; ASA: aspirin; PTCA: percutaneous transluminal coronary angioplasty; BMS: bare metal stent; DES: drug-eluting stent; MI: myocardial infarction; ST: stent thrombosis.

\section{Perioperative management of AP agents}

The general approach to managing patients on antiplatelet agents preoperatively is similar to that for Anticoagulant agents. Defining the fine balance between ischemic and bleeding risk remains a challenge in patients with coronary stents undergoing surgery treated with antiplatelet therapy. The risk of recurrent ischemic events for patients discontinuing or not adhering to aspirin treatment has been suggested to increase 3 -fold ${ }^{12}$. Stent thrombosis is a serious complication that commonly presents with death or a significant nonfatal myocardial infarction. Ischemic events are more common after the premature discontinuation of a second antiplatelet agent such as clopidogrel and it can be explained by "withdrawal of protection"12. This can be accomplished by avoiding drug-eluting stents whenever possible, especially in patients with known poor adherence to medical therapy and with any anticipated surgeries.

Figure 1 highlights a suggested algorithm for perioperative management of patients on AP agents, as presented by Di Minno et al in 2013

Low risk situations are defined as $>14$ days post balloon angioplasty, > 6 weeks post bare metal stent and $>6$ months post drug eluting stent. If the patients is at low risk of major adverse cardiac events and have a low risk of bleeding, then the dual AP agents can be discontinued, but it is preferred if Aspirin is continued.

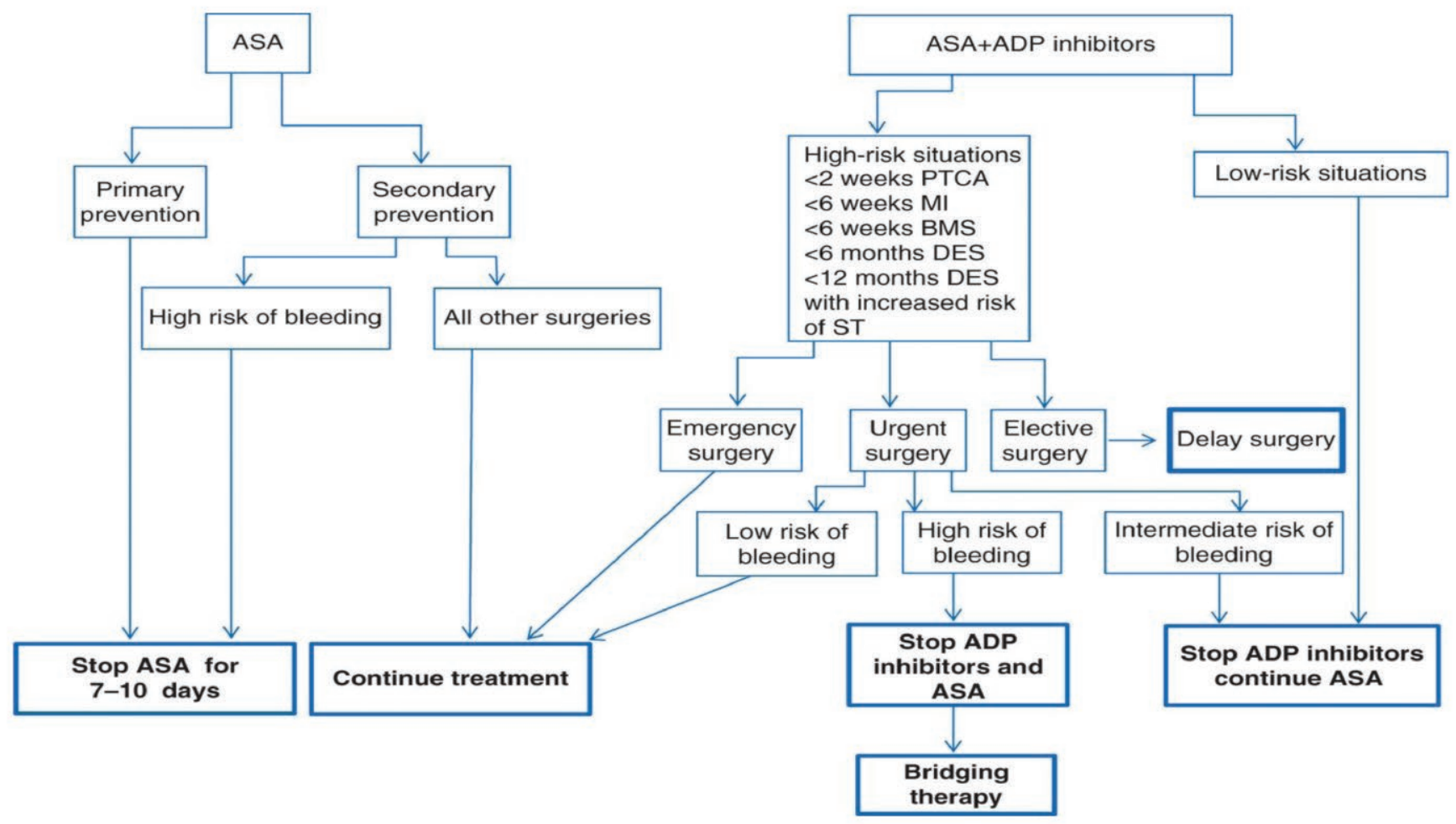


The Medicine Forum, Vol. 17 [2016], Art. 21

\section{Case 6: Dual AP agents post DES}

68 -year-old man with a sirolimus-eluting coronary stent inserted 4 months ago following NSTEMI. Now requires surgery for removal of a parotid neoplasm (adenocarcinoma $\rightarrow$ Urgent surgery

ASA, $81 \mathrm{mg}+$ clopidogrel, 75 mg daily

CABG 8 years ago, Hypertension, Type 2 diabetes

$\rightarrow$ high risk of thrombosis and low risk of bleeding

Dr. Marhefka: In patients who had an MI, the observational data shows that up to a year there are complications with non-cardiac surgeries. We don't use sirolimus stents anymore, which are considered first generation stents. But if we had a patient with that such as in our case, and is beyond a year, we would be comfortable stopping the second AP agents but not the aspirin (ASA). Therefore, with a first generation stent, we would continue the surgery on ASA. We would push for surgery on double AP agents if it is 4 months post stenting, or delay the surgery to at least 1 year and then do the surgery only on ASA. If the case is of a second-generation stent, there is data with xerolimus and everolimus that showed evidence of 3 months of dual AP therapy is enough, but keep in mind that an NSTEMI was recent.

Dr. DeCaro: Our surgeons have reached a certain comfort level performing surgeries on dual AP agents.

Dr. Nagalla: One option might be to stop the AP agent, bridge with heparin and then restart the agent post-op.

Audience: This area needs more evidence to know how to manage AP agents. We have to respect the surgeon's preferences, even if it is anecdotal or cultural rather than evidence based since they are the ones performing the surgery.

\section{Suggested management plan for case 6 :}

- Optimal to delay surgery to at least 6 months after DES

- Since it is urgent due to active malignancy, and low risk of bleed, will proceed with surgery on DAPT without interruption.

\section{CONCLUSION}

Managing patients on blood thinners perioperatively can be very challenging and an optimal approach is an interdisciplinary discussion weighing the benefits to risks of stopping the agent or bridging whenever necessary. Patients should be fully informed of the risks of thrombosis versus bleeding when consenting to the plan. The available consensus statements have been serving as "guidelines", but as our expert panelists have reminded us, they are class 2 recommendations formed by expert consensus. It is important to take these recommendations in the context of every patient for an individualized approach to treatment.

\section{REFERENCES}

1. January CT, Wann LS, Alpert JS, Calkins H, Cigarroa JE, Cleveland JC Jr, et al. 2014 AHA/ACC/HRS guideline for the management of patients with atrial fibrillation. Journal of the American College of Cardiology. 2014;64(21):2246-80.

2. Mozaffarian D, Benjamin EJ, Go AS, Arnett DK, Blaha MJ, Cushman M, et al. Heart disease and stroke statistics-2015 update: a report from the American Heart Association. Circulation. 2015;131:e29-e322

3. Berger PB, Kleiman NS, Pencina MJ, Hsieh WH, Steinhubl SR, Jeremias A, SonelA, Browne K, Barseness G, Cohen DJ; EVENT Investigators. Frequency of major noncardiac surgery and subsequent adverse events in the year after drug-eluting stent placement results from the EVENT (Evaluation of Drug-Eluting Stents and Ischemic Events) Registry. JACC Cardiovasc Interv. 2010;3:920-927.

4. Douketis JD, Spyropoulos AC, Spencer FA, et al. Perioperative management of antithrombotic therapy: Antithrombotic Therapy and Prevention of Thrombosis, 9th ed: American College of Chest Physicians Evidence-Based Clinical Practice Guidelines. Chest 2012; 141:e3265-e350S

5. Mehran R, Rao SV, Bhatt DL, et al. Standardized bleeding definitions for cardiovascular clinical trials: a consensus report from the Bleeding Academic Research Consortium. Circulation 2011; 123:2736-2747.

6. Spyropoulos AC and Douketis JD. How I treat anticoagulated patients undergoing an elective procedure or surgery. Blood 2012; 120:2954-2962.

7. Douketis J. Perioperative management of patients who are receiving warfarin therapy: an evidence-based and practical approach. Blood. 2011, 117(19): 5044-5049.

8. Hirsh J, Bauer KA, Donati MB, et al. Parenteral anticoagulants: American College of Chest Physicians Evidence-Based Clinical Practice Guidelines (8th Edition). Chest 2008; 133:141S-1595

9. O'Donnell MJ, et al. Brief communication: Preoperative anticoagulation activity after bridging low-molecular-weight heparin for temporary interruption of warfarin. Ann Intern med 2007;146(3):184-187.

10. Horlocker T, Wedel D, Rowlingson J. et al. Regional Anesthesia in the Patient Receiving Antithrombotic or Thrombolytic Therapy: American Society of Regional Anesthesia and Pain Medicine Evidence-Based Guidelines (Third Edition). Regional Anesthesia \& Pain Medicine 2010;35(1):64-101

11. Anticoagulation 4th Edition (4th ASRA practice advisory for regional anesthesia) Retrieved March 30m 2016, from https://www.asra.com/advisory-guidelines/ article/1/anticoagulation-3rd-edition

12. Capodanno D and Angiolillo DJ. Management of Antiplatelet Therapy in Patients With Coronary Artery Disease Requiring Cardiac and Noncardiac Surgery Circulation 2013;128:2785-2798.

13. Di Minno MN, Milone M, Mastronardi P, et al. Perioperative handling of antiplatelet drugs. A critical appraisal. Curr Drug Targets 2013; 14: 880-888 